\title{
Risk Estimation and Value-of-Information Analysis for Three Proposed Genetic Screening Programs for Chronic Beryllium Disease Prevention
}

\author{
Scott M. Bartell, ${ }^{1,2}$ Rafael A. Ponce, ${ }^{1,2}$ Timothy K. Takaro, ${ }^{2,3}$ Richard O. Zerbe, ${ }^{4}$ \\ Gilbert S. Omenn, ${ }^{5}$ and Elaine M. Faustman ${ }^{1,2,6}$
}

\begin{abstract}
Genetic differences (polymorphisms) among members of a population are thought to influence susceptibility to various environmental exposures. In practice, however, this information is rarely incorporated into quantitative risk assessment and risk management. We describe an analytic framework for predicting the risk reduction and value-of-information (VOI) resulting from specific risk management applications of genetic biomarkers, and we apply the framework to the example of occupational chronic beryllium disease (CBD), an immune-mediated pulmonary granulomatous disease. One described Human Leukocyte Antigen gene variant, HLA-DP $\beta 1 * 0201$, contains a substitution of glutamate for lysine at position 69 that appears to have high sensitivity ( $\sim 94 \%$ ) but low specificity ( $\sim 70 \%$ ) with respect to CBD among individuals occupationally exposed to respirable beryllium. The expected postintervention CBD prevalence rates for using the genetic variant (1) as a required job placement screen, (2) as a medical screen for semiannual in place of annual lymphocyte proliferation testing, or (3) as a voluntary job placement screen are $0.08 \%, 0.8 \%$, and $0.6 \%$, respectively, in a hypothetical cohort with $1 \%$ baseline CBD prevalence. VOI analysis is used to examine the reduction in total social cost, calculated as the net value of disease reduction and financial expenditures, expected for proposed CBD intervention programs based on the genetic susceptibility test. For the example cohort, the expected net VOI per beryllium worker for genetically based testing and intervention is $\$ 13,000, \$ 1,800$, and $\$ 5,100$, respectively, based on a health valuation of $\$ 1.45$ million per $\mathrm{CBD}$ case avoided. VOI results for alternative $\mathrm{CBD}$ valuations are also presented. Despite large parameter uncertainty, probabilistic analysis predicts generally positive utility for each of the three evaluated programs when avoidance of a CBD case is valued at $\$ 1$ million or higher. Although the utility of a proposed risk management program may be evaluated solely in terms of risk reduction and financial costs, decisions about genetic testing and program implementation must also consider serious social, legal, and ethical factors.
\end{abstract}

KEY WORDS: Value-of-information, beryllium, polymorphism, occupational health, epidemiology

${ }^{1}$ Department of Environmental Health and Institute for Risk Analysis and Risk Communication, University of Washington, 4225 Roosevelt Way NE \#100, Seattle, WA 98105-6099.

${ }^{2}$ Consortium for Risk Evaluation with Stakeholder Participation, University of Washington, Seattle, WA.

${ }^{3}$ Department of Medicine, University of Washington, Seattle, WA.

${ }^{4}$ Graduate School of Public Affairs, University of Washington, Seattle, WA.

${ }^{5}$ School of Medicine and School of Public Health, University of Michigan, Ann Arbor, MI.

${ }^{6}$ Center for Ecogenetics and Environmental Health, University of Washington, Seattle, WA; corresponding author.

\section{INTRODUCTION}

Biomarkers have the potential to refine many toxicological risk assessment model parameters, thereby reducing uncertainty regarding individual and population risk. Although exposure biomarkers have been used in risk assessment for some time, and clinical effect biomarkers have long been part of medical practice, public health practitioners are struggling 
with the practical application of biomarkers of susceptibility or preclinical effect. ${ }^{(1)}$ Genetic biomarkers are particularly underused in legitimate disease prevention, partly because of the controversial nature of their use and partly because of complexities in judging their practical utility.

In this article, we use risk assessment techniques to estimate the potential disease reduction among beryllium workers from the proposed use of relevant genetic information. In particular, we estimate the impact of proposed screening programs and heightened medical surveillance based on screening beryllium workers for a genetic polymorphism at position 69 (Glu-69) of a Human Leukocyte Antigen allele, HLA-DP $\beta 1$, which is involved in antigen recognition. ${ }^{(2)}$ We further apply basic benefit-cost analysis ${ }^{(3-5)}$ and value-of-information methods $s^{(4,5)}$ to assess the net social benefits derived from using individual genetic information to identify and protect susceptible individuals. Clearly the usefulness of genetic information depends on a number of legal, ethical, and social factors. We briefly address the implications of these factors, as well as the costs and occupational health implications of genetic screening using the Glu-69 test. Model calculations are conducted by using probability distributions to represent uncertainty.

\section{BACKGROUND}

Beryllium is a rare metal primarily used in the ceramics, aircraft, and nuclear industries during the last half-century, though recent reports indicate new applications in consumer products such as golf clubs and mountain bicycles. ${ }^{(6)}$ Respirable beryllium particles are toxic to humans; they cause pneumonitis (acute beryllium disease) at high exposures and chronic beryllium disease (CBD) at lower exposures. CBD is generally characterized by diffuse interstitial infiltration, alveolitis, and fibrosis and often involves development of granulomas. Beryllium-induced granulomas are histologically indistinguishable from those found in sarcoidosis, tuberculosis, and Crohn's disease, ${ }^{(7)}$ or from granulomas caused by aluminum and titanium. ${ }^{(8)}$ Though beryllium diseases including cancer and acute beryllium disease are generally associated with pulmonary dysfunction, other organs, including the lymphatic system, heart, skin, liver, and spleen, may be affected. ${ }^{(7,9)}$

After 1949, when an exposure standard of $2 \mu \mathrm{g} /$ $\mathrm{m}^{3}$ in air was implemented by the U.S. Atomic Energy Commission, acute beryllium disease was essentially eliminated in the U.S. but the incidence of CBD continued unabated. The Occupational Safety and Health Administration (OSHA) later adopted and currently enforces the $2 \mu \mathrm{g} / \mathrm{m}^{3}$ standard. CBD, however, is still prevalent among workers exposed to beryllium dusts, even at exposures generally below $2 \mu \mathrm{g} / \mathrm{m}^{3} .^{(10)}$

The overall prevalence rate of CBD among individuals known to have worked in beryllium-contaminated areas ranges from about $1 \%$ to $5 \% .{ }^{(11)}$ Among beryllium workers routinely engaged in certain work tasks, such as machining and ceramics dry pressing, CBD prevalence is as high as $10 \%$ to $20 \%$, $^{(10,12)}$ though associated median daily weighted average beryllium air concentrations were below the OSHA standard. Though the "beryllium worker" characterization varies from study to study, most studies now include administrators, engineers, janitors, and other nonmachinists if they are known to have regularly been present in buildings with ongoing or previous beryllium use or potential exposure. Cases of CBD have been identified among these individuals ${ }^{(10,12)}$ as well as among workers' spouses presumably exposed through contaminated clothing, ${ }^{(13)}$ despite their lack of direct involvement in high-risk activities.

A beryllium worker health surveillance program was instituted at the beginning of the decade at the U.S. Department of Energy (DOE) Oak Ridge Y-12 Plant and the Rocky Flats Environmental Technology Site. The program now includes dozens of other DOE sites where beryllium was used and has identified approximately 9,000 workers with potential previous occupational beryllium exposure. ${ }^{(14)}$ Despite exposure reductions to levels generally at or below the OSHA standard, roughly $1 \%$ of beryllium-exposed DOE workers have developed CBD. ${ }^{(14)}$

The similar pathogenesis of CBD to other pulmonary granulomatous diseases, including sarcoidosis, and available animal-based experimental data suggest that susceptibility to CBD involves a genetic component. Although a number of genetic polymorphisms have been investigated to identify potential associations with $\mathrm{CBD}$, the presence of a glutamate for lysine substitution at position 69 of HLA-DP $\beta 1$ $($ Glu-69+) has been found to have the highest correlation with $\mathrm{CBD},{ }^{(2)}$ and has been suggested as a potential screening tool for occupational CBD risk management. ${ }^{(15)}$ Human Leukocyte Antigen (HLA) genes are a group of genes similar to Major Histocompatability Complex genes found in other species. Certain HLA genes located in the D-region on the short arm of chromosome 6 (DP $\alpha 1, \mathrm{DP} \alpha 2, \mathrm{DP} \beta 1$, and DP $\beta 2)$ produce tetrameric proteins involved in antigen rec- 
ognition that bind to endocytosed fragments such as beryllium. Polymorphisms in the beta chains are frequent and are suspect contributors to immunemediated metal responses. ${ }^{(2)}$

Another screening biomarker is the beryllium lymphocyte proliferation test (LPT), for which a positive result indicates immunologic sensitization to beryllium and heightened susceptibility to CBD. This in vitro assay consists of an evaluation of the proliferative response of isolated lymphoctyes to the presence of beryllium. A positive LPT result is considered a sign of immunologic sensitization to beryllium and is usually followed by CBD. ${ }^{(16)}$ At DOE sites, beryllium workers are currently tested with the LPT every 1 to 3 years. Sensitized workers (as indicated by a confirmed positive LPT) are reassigned to jobs without beryllium exposure and followed up with additional medical monitoring for CBD. ${ }^{(14)}$

\section{METHODS}

In the present analysis we use risk assessment techniques to assess the impact of using the Glu-69 test as a placement or medical screen for occupational beryllium exposure. One proposed intervention is the removal of genetically susceptible (i.e., Glu-69 $9^{+}$workers from beryllium-contaminated areas, which might require job reassignment. This intervention could be required or strictly voluntary. Another option is more aggressive medical monitoring for genetically susceptible individuals. This might consist of more frequent LPT, physical examinations, $\mathrm{X}$-rays, or other clinical screens. Heightened medical surveillance of Glu- $69^{+}$individuals might be a reasonable intervention strategy for CBD because individuals in the early stages of disease would be identified more quickly, thereby allowing rapid removal from further exposure to beryllium and earlier treatment where warranted.

We evaluate three specific examples of Glu69-based risk management options: (1) required screening of workers for job placement in beryllium-contaminated areas, (2) increased frequency of LPT monitoring for Glu- $69^{+}$beryllium-exposed workers from annual to semiannual testing, and (3) voluntary genetic testing and informed decison making by workers with potential beryllium exposure. The expected reduction in CBD risk and the implementation cost per person are estimated for each of these three genetic interventions by using distributional analysis. We generated 20,000 simple random samples for each variable during Monte Carlo simulation.

\subsection{CBD Risk Model}

For risk management using binary susceptibility biomarkers such as Glu-69, the expected postintervention disease risk $\left(r_{p}\right)$ is the populationweighted average of the CBD risk among those workers testing negative for the biomarker $\left(\mathrm{Glu}-69^{-}\right)$ and the post-intervention CBD risk among workers testing positive for the biomarker:

$r_{p}=\operatorname{Pr}\left[\right.$ Glu- $\left.69^{-}\right] \operatorname{Pr}\left[\mathrm{CBD}^{+} \mid \mathrm{Glu}-69^{-}\right.$, no intervention $]$

$$
+\operatorname{Pr}\left[\text { Glu- } 69^{+}\right] \operatorname{Pr}\left[\mathrm{CBD}^{+} \mid \text {Glu- } 69^{+} \text {, intervention }\right]
$$

Conditional disease probabilities are often written as sensitivity, specificity, or predictive value (see Appendix A). We substitute positive predictive value $\left(P V^{+}\right)$, negative predictive value $\left(P V^{-}\right)$, and intervention efficacy $(e)$ into Eq. 1 to yield the following:

$$
\begin{aligned}
r_{p}= & \operatorname{Pr}[\text { Glu-69- }]\left(1-P V^{-}\right) \\
& +\operatorname{Pr}\left[\text { Glu-69 } 9^{+}\right](1-e) P V^{+}
\end{aligned}
$$

Preplacement occupational screening is one genetically based risk management option. This type of risk management program relies on changing the proportion of workers with genetic susceptibility, rather than reducing the risks to existing workers. If $f$ is the prevalence of Glu- $69^{+}$among job applicants and $w$ represents the likelihood of a Glu- $69^{+}$applicant being removed through screening, the post-screening fraction of workers with the Glu-69+ $9^{+}$substitution is expected to be $f(1-w)+f(1-w) f w+\mathrm{f}(1-w)(f w)^{2}+$ $f(1-w)(f w)^{3}+\ldots=f(1-w) /(1-f w)$. The remaining fraction, $(1-f) /(1-f w)$, indicates the postscreening proportion of Glu- $69^{-}$workers. Eq. 2 can therefore be rewritten as:

$$
r_{p}=\frac{1-f}{1-f w}\left(1-P V^{-}\right)+\frac{f(1-w)}{1-f w}(1-e) P V^{+}
$$

We use Eq. 3 to predict the risk reduction attained through various genetically based risk management programs. Because $P V^{+}$and $P V^{-}$are dependent on the baseline prevalence of disease, they can be directly observed only in cohort studies. However, if the baseline prevalence is known or assumed, the sensitivity and specificity from case-control studies may be used to estimate predictive values as described by Hennekens and Buring. ${ }^{(17)}$ We assume that reported Glu-69 sensitivity and specificity rates for 
CBD are constant with respect to baseline prevalence and use the three parameters to estimate $P V^{+}$ and $P V^{-}$as described in the Appendix.

\subsection{Glu-69 VOI Model}

Analysis of the expected costs and benefits associated with a risk management option provides information that is useful for rational decison making. Valueof-information (VOI) analysis is used to estimate the impact of new data on the net social costs or opportunity loss associated with a decision. . $5,18,19)$ We have chosen to evaluate VOI for each Glu-69-based risk management program in the context of its predicted effect on expected total social cost (TSC). The TSC expresses the net value of the health impacts and financial costs associated with a health risk management alternative:

$$
T S C=F C+r_{p} B_{d}
$$

where $F C$ is the per capita financial cost of program implementation, including administrative costs, biomarker sampling and laboratory analysis costs, and any other expenses, and $B_{d}$ is the health cost associated with each case of CBD. Broader social or legal costs associated with each genetic intervention are not included in our evaluation of TSC but could be added to Eq. 4 if desired. ${ }^{(5)}$ The cost per beryllium worker of voluntary screening is related to the cost of genetic testing for each job applicant $\left(C_{\text {genotype }}\right)$ and the cost of genetic counseling for each Glu- $69^{+}$job applicant $\left(C_{\text {counsel }}\right)$ by the following equation:

$$
F C=\frac{C_{\text {genotype }}+f C_{\text {counsel }}}{1-f w}
$$

We set $C_{\text {counsel }}$ to 0 for required preplacement screening because we assume that genetic counseling would not be necessary in this case. Eq. 5 assumes that all job applicants will undergo genetic testing but only Glu- $69^{+}$individuals will require counseling. If all Glu-69- and Glu- $69^{+}$individuals are to be counseled, $f$ is removed from the numerator of Eq. 5. In the baseline case, where no susceptibility biomarker information is collected and no control strategy is implemented, $F C=0$ and $r_{p}=p$.

The cost per beryllium worker of a genetically targeted LPT frequency increase is the sum of the cost of genetic testing for each worker $\left(C_{\text {genotype }}\right)$ and the cost of additional LPTs for each Glu-69+ $9^{+}$worker $\left(C_{L P T}\right)$ :

$$
F C=C_{\text {genotype }}+f C_{L P T}
$$

No adjustments are made for costs associated with slightly more frequent hiring associated with the LPT based genetic intervention, though this is an expected result of earlier detection and removal of sensitized workers. The effect on costs is assumed to be negligible for the case presented here.

VOI expresses the predicted degree to which a Glu-69-based CBD intervention program mitigates total social impacts:

$$
V O I=T S C_{\text {baseline }}-T S C_{\text {option }}
$$

where $T S C_{\text {option }}$ is the total social cost for the specified risk management option and $T S C_{\text {baseline }}$ is the total social cost for the baseline option (i.e., no testing for Glu-69). The most cost-effective risk management option is that which maximizes VOI.

\subsection{Characterization of Input Variables}

Because many of the parameters used to calculate VOI are uncertain, we apply probabilistic uncertainty analysis using Monte Carlo simulation techniques. ${ }^{(20)}$ In earlier work, we assigned point estimates and plausible bounds for each parameter and used them to calculate a best estimate and plausible range of VOI for the Glu-69 test in each risk management program. ${ }^{(21)}$ Here we estimate the likelihood of all possible values for most model parameters by using subjective probability distributions (Table I) in combination with fixed prevalence rates and fixed CBD avoidance values. The basis for each of these estimates is described in the following section.

Variables treated as uncertain in this analysis include the sensitivity and specificity of the Glu-69 test, the cost per LPT and cost per Glu-69 test, the impact of early LPT detection of sensitization on CBD progression, and the proportion of workers in the voluntary screening program who decide to remove themselves from exposure if test results indicate genetic susceptibility (i.e., Glu- $69^{+}$).

\subsubsection{CBD Prevalence; Sensitivity and Specificity to Glu-69}

The value of Glu-69 testing, or any other type of genetic testing, depends on the prevalence of disease in the target population. The prevalence of CBD among beryllium workers is reported to be about $1 \%$ to $16 \%$ depending upon specific job task, ${ }^{(16)}$ and $1 \%$ to $5 \%$ averaged among all beryllium workers. ${ }^{(22)}$ We repeat the CBD risk and VOI calculations over a range 
Table I. Input Parameters for Glu-69 Value-of-Information Model

\begin{tabular}{|c|c|c|c|}
\hline Parameter & Description & Value $^{a}$ & Confidence $^{b}$ \\
\hline$S n$ & Sensitivity of Glu-69 for CBD prediction & $\beta(33,2)$ & High \\
\hline$S p$ & Specificity of Glu- 69 for CBD prediction & $\beta(32,14)$ & High \\
\hline$B_{d}$ & Statistical value of CBD (\$million/case) & Various (see Table III) & Low \\
\hline$C_{\text {genotype }}$ & Cost of Glu-69 testing (\$/applicant) & $\mathrm{T}(100,150,500)$ & Medium \\
\hline$C_{L P T}$ & Present value of costs of increased LPT frequency $\left(\$ / \mathrm{Glu}-69^{+}\right.$worker) & $\mathrm{U}(700,2,200)$ & Medium \\
\hline$C_{\text {counsel }}$ & Cost of genetic counseling ( $\$ /$ Glu- $69^{+}$applicant $)$ & $\mathrm{T}(50,100,200)$ & Medium \\
\hline$w_{\text {screen }}$ & Removal probability for a Glu- $69^{+}$worker for required screening & $100 \%$ & High \\
\hline$w_{L P T}$ & Removal probability for a Glu- $69^{+}$worker for increased LPT frequency & $0 \%$ & Medium \\
\hline$w_{\text {volunteer }}$ & Removal probability for a Glu- $69^{+}$worker for voluntary screening & $\mathrm{U}(0 \%, 100 \%)$ & Medium \\
\hline$e_{\text {screen }}$ & Risk reduction efficacy (for a Glu- $69^{+}$worker) of required screening & $0 \%$ & High \\
\hline$e_{L P T}$ & Risk reduction efficacy (for a Glu- $69^{+}$worker) of increased LPT frequency & $\mathrm{T}(0 \%, 5 \%, 50 \%)$ & Low \\
\hline$e_{\text {volunteer }}$ & Risk reduction efficacy (for a Glu- $69^{+}$worker) of voluntary screening & $0 \%$ & Medium \\
\hline
\end{tabular}

${ }^{a} \beta(v, w)$ indicates a beta distribution, $\mathrm{U}(l, u)$ indicates a uniform distribution, and $\mathrm{T}(l, m, u)$ indicates a triangular distribution. Values are assigned on the basis of empirical evidence, theoretical models, and judgment.

${ }^{b}$ Confidence indicates the assessors' level of comfort that the distribution shape and parameters assigned to the given parameter are appropriate given the existing state of knowledge.

of potential baseline prevalence rates. Thus, for any specific beryllium-exposed worker cohort, the predicted risk reduction and VOI can be determined by using the baseline CBD prevalence.

The first study to correlate HLA polymorphisms with CBD status found that $97 \%$ of CBD-positive beryllium workers had Glu- $69^{+}(n=33)$ but that only $70 \%$ of CBD negative beryllium workers were Glu$\left.69^{-}(n=44)\right)^{(2)}$ A more recent cohort study by the same principal investigator found Glu-69 sensitivity and specificity rates of $83 \%$ and $70 \%$, respectively, among 6 CBD cases and 121 controls. ${ }^{(23)}$ Because the cohort study had very few cases of disease, the lower estimated sensitivity rate derived from that study has a very wide confidence interval and is statistically indistinguishable from the estimates derived from the original study. We chose to use the casecontrol study as a basis for selecting the sensitivity and specificity rates for our model. On the basis of the conditional likelihood of the sensitivity and specificity rates given the observed binomial variation, we represent the uncertainty in those rates with beta distributions (Table I).

\subsubsection{Value of CBD Avoidance; Cost of Glu-69 Screening and LPT Testing}

$B_{d}$ indicates the economic preference for avoiding a single case of CBD and is typically determined by using "cost of illness" (COI) approaches, "willingness to pay" (WTP) measures, or other methods. ${ }^{(24)}$ COI estimates are obtained by disaggregation of di- rect and indirect costs (e.g., health care costs and lost wages). COI estimates underestimate the societal value of disease avoidance because they place no value on lost time other than for wage losses. Though they also fail to value pain and suffering associated with disease, COI techniques are useful for initially estimating the value of disease avoidance. WTP approaches implicitly include pain and suffering as well as the COI.

In practice, health valuation estimates vary considerably according to the methods and assumptions chosen. The choice of valuation method and discount rate strongly influence the final valuation. We select a variety of estimates based on different approaches and repeat the risk and VOI calculations for each estimate.

We obtain a low estimate of the COI for CBD based on the medical cost savings per CBD case avoided according to a DOE economic analysis. ${ }^{(25)}$ The mean valuation for the four potential treatment scenarios in that analysis is $\$ 12,200$. This valuation uses a discount rate of $7 \%$ and assumes that workers are diagnosed with CBD at age 40 and live to age 70 . A second COI estimate ${ }^{(21)}$ is based on reported treatment costs and lost wages for general respiratory diseases. For this estimate we assumed 45 years would be spent with reduced activity and extended the daily respiratory disease costs throughout that entire period without discounting to obtain an expected value of $\$ 1,450,000$ per case of CBD avoided. With a 5-year latency and a $7 \%$ discount rate, this value is reduced to approximately $\$ 290,000$ per CBD case. We evaluate the results by using both the discounted and nondiscounted values (Table I). 
No WTP estimates are currently available for CBD. However, most individuals diagnosed with CBD will die from beryllium-related disease. ${ }^{(25)} \mathrm{Be}-$ cause of a combination of high mortality and longterm morbidity, applying life valuations to CBD may be appropriate. Estimates for the statistical value of life run as high as $\$ 16,300,000 .{ }^{(26)}$ We include that figure as the highest value in our analysis, without discounting for latency.

We estimate that laboratory analysis for Glu-69 will cost $\$ 100$ per sample. ${ }^{(21)}$ Allowing an extra $\$ 50$ per sample for sample collection and shipment, we estimate a total cost for Glu-69 testing of \$150/sample and assign bounds of $\$ 100$ and $\$ 500$ to allow for a large degree of error. This parameter $\left(C_{\text {genotype }}\right)$ is represented by using a triangular distribution (Table I).

Voluntary preplacement screening would require some counseling for applicants testing Glu- $69^{+}$. We suggest at least an hour's worth of counseling for each individual along with the production of written materials for the applicant to take home. Our best estimate of this cost is $\$ 100$ per Glu- $69^{+}$applicant. We assign a triangular distribution allowing possible values in the range of half to double the primary estimate (Table I).

The present value of the costs from 50 years of additional LPTs in one individual (one extra test per year over a lifetime) is estimated to be $\$ 2,200$ per Glu- $69^{+}$individual using a 7\% discount rate and $\$ 231$ per LPT. ${ }^{(25)}$ If a Glu- $69^{+}$individual develops a positive LPT during the fifth year of exposure and is immediately replaced by a Glu-69- individual, the discounted cost of additional LPTs is about $\$ 700$. The true value is likely to lie somewhere between these two estimates, so we represent $C_{L P T}$ by using a uniform distribution (Table I).

\subsubsection{Predicted Risk Reduction Efficacy and Preplacement Removal Probability}

The efficacy of the proposed risk management programs reflects how well they target and change the behavior, exposure, or medical treatment of Glu- $69^{+}$individuals. In a required screening program, essentially $100 \%$ of Glu- $69^{+}$beryllium work applicants would be replaced by Glu-69- individuals. With voluntary screening participation might be somewhat lower, depending on the proportion of workers consenting to a susceptibility test and deciding to remove themselves from exposure when test results are positive. Because participation in the voluntary screening program is highly uncertain, we assign a uniform distribution allowing for anywhere between $0 \%$ and $100 \%$ removal likelihood for Glu$69^{+}$applicants.

Medical or industrial hygiene intervention could reduce disease rates through a different use of genetic information. Rather than screening out genetically susceptible applicants before they are exposed to beryllium, this type of intervention seeks to reduce the disease risk or severity through targeted exposure mitigation or heightened medical monitoring. We assume that increasing LPT frequency would reduce CBD risk by removing workers from beryllium exposure earlier in the process of disease development. No known evidence supports or contradicts the assumed benefits of early detection of beryllium sensitization, but it is reasonable based on other immunemediated disease models to assume that CBD risk increases with exposure duration. Assuming a uniform likelihood of CBD development in the time interval between LPTs, increasing the LPT frequency from once a year to twice a year would reduce the average beryllium exposure duration by 3 months for sensitized individuals. What effect if any this would have on disease progression is unclear, but some evidence indicates that CBD risk increases with exposure duration. ${ }^{(27)}$ Hypothesizing that the prevalence of disease might be linearly related to exposure duration for Glu- $69^{+}$individuals, detection and removal from exposure 3 months early in a cohort with a 5-year latency would reduce CBD prevalence by $5 \%$ among individuals in that cohort assuming they are replaced by workers who do not become sensitized. Alternatively, the prevalence of disease could be proportional to the postsensitization exposure duration, in which case early detection would decrease the CBD prevalence by $50 \%$. Clearly a number of other models could be formed, including the possibility that early detection does not impact the likelihood of CBD development. We assign a triangular distribution to this parameter, with bounds of $0 \%$ and $50 \%$ and a mode of $5 \%$ (Table I).

\section{RESULTS}

Predicted postintervention CBD risks are shown in Table II. The risk estimates for $1 \%$ baseline prevalence are graphed in Figure 1 for visual comparison. The postintervention CBD risk is nearly linear with baseline prevalence in the range of interest, so Figure 1 can be used to visualize the approximate postintervention 
Table II. Predicted Postintervention CBD Risk by Baseline Prevalence and Intervention Type

\begin{tabular}{|c|c|c|}
\hline $\begin{array}{l}\text { Baseline } \\
\text { prevalence }\end{array}$ & Intervention type & $\begin{array}{l}\text { Postintervention } \\
\text { CBD risk }\end{array}$ \\
\hline $0.1 \%$ & Required screen & $0.008 \%(0.001 \%, 0.020 \%)^{a}$ \\
\hline $0.1 \%$ & $\begin{array}{l}\text { Increased LPT } \\
\text { frequency }\end{array}$ & $0.08 \%(0.06 \%, 0.10 \%)$ \\
\hline $0.1 \%$ & Voluntary screen & $0.06 \%(0.02 \%, 0.10 \%)$ \\
\hline $1 \%$ & Required screen & $0.08 \%(0.02 \%, 0.20 \%)$ \\
\hline $1 \%$ & $\begin{array}{l}\text { Increased LPT } \\
\text { frequency }\end{array}$ & $0.8 \%(0.6 \%, 1.0 \%)$ \\
\hline $1 \%$ & Voluntary screen & $0.6 \%(0.2 \%, 1.0 \%)$ \\
\hline $10 \%$ & Required screen & $0.9 \%(0.2 \%, 2.1 \%)$ \\
\hline $10 \%$ & $\begin{array}{l}\text { Increased LPT } \\
\text { frequency }\end{array}$ & $8.3 \%(6.3 \%, 9.7 \%)$ \\
\hline $10 \%$ & Voluntary screen & $6.5 \%(1.6 \%, 9.7 \%)$ \\
\hline
\end{tabular}

${ }^{a}$ First value indicates expected value of CBD risk; range in parentheses indicates $90 \%$ simulation interval as defined by the 5 th and 95th percentiles of Monte Carlo simulation results. These values depend on hypothetical models and subjective parameter estimates shown in Table I.

CBD risks at any baseline prevalence rate by rescaling the $\mathrm{y}$-axis from 0 to $p$. In a cohort with $1 \%$ baseline CBD prevalence, the expected postintervention prevalence rate for using the Glu-69 test as a required job placement screen is $0.08 \%$. A voluntary screening program in the same cohort would be expected to reduce CBD prevalence to $0.6 \%$, but this value is highly sensitive to the participation rate. Use of the genetic

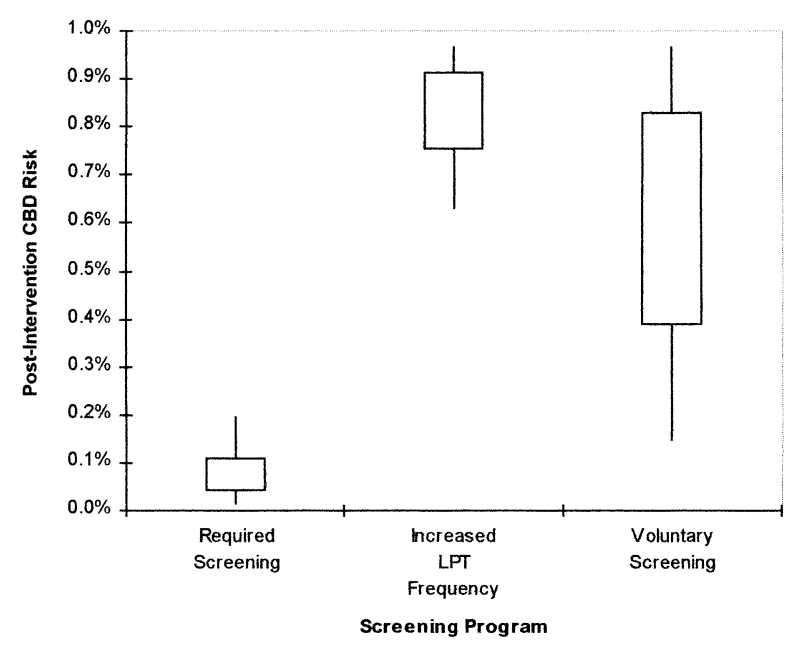

Fig. 1. Simulated post-intervention CBD risk at $1 \%$ baseline prevalence. $50 \%$ simulation intervals are displayed as boxes and $90 \%$ simulation intervals are shown as outer lines. These values depend on hypothetical models and subjective parameter estimates shown in Table I. variant as a medical screen for semiannual in place of annual lymphocyte proliferation testing yields a $0.8 \%$ mean predicted postintervention prevalence for the example cohort. The $90 \%$ simulation intervals, defined by the 5th and 95th percentiles of Monte Carlo simulation output, are $(0.02 \%, 0.2 \%)$ for required screening, $(0.6 \%, 1 \%)$ for increased LPT frequency, and $(0.2 \%, 1 \%)$ for voluntary screening. These predicted postintervention risks and the other analytical results depend heavily on the parameter estimates in Table I and the underlying model assumptions.

Net expected VOI per beryllium worker is estimated across a range of baseline prevalence values and CBD avoidance valuations (Table III). For most current occupational prevalence rates and health valuations exceeding $\$ 1.45$ million, the mean predicted VOI exceeds zero, indicating an expected net social gain for using the susceptibility biomarker in any of the three proposed strategies. For example, at $10 \%$ baseline CBD prevalence and $\$ 1.45$ million per case of CBD, the expected net per capita VOI for Glu-69 in required or voluntary screening increases to $\$ 130,000$ or $\$ 51,000$, respectively. However, at low baseline CBD prevalence rates and low health valuations, the expected VOI for the three Glu- $69^{+}$based programs is negative, which indicates that the program costs are expected to outweigh the benefits of CBD reduction.

The baseline CBD prevalence and CBD valuation for which the expected VOI is zero, or "cutpoints," are shown as lines in Figure 2. Each Glu-69based risk management program is expected to be cost effective only when the baseline prevalence and selected health valuation produce a point to the right of the respective cutpoint line. These graphs could be expanded by adding VOI endpoints other than zero or displaying cutpoints based on simulation bounds (e.g., 5th and 95th percentiles) to indicate the effects of uncertainty.

Sensitivity analysis was conducted for the three risk management programs at $1 \%$ baseline prevalence and $\$ 1.45$ million per CBD case avoided. Note that $p$ and $B_{d}$ have the strongest influence by far on the model results and the sensitivity analysis indicates the relative importance of the remaining variables. Contribution to variance estimates based on Spearman's rank correlation indicate that for required screening about $94 \%$ of the VOI simulation variance is associated with the sensitivity rate of CBD to Glu-69 $(S n)$; the specificity rate $(S p)$ and Glu-69 financial cost $\left(C_{\text {genotype }}\right)$ each contribute about $3 \%$ of the 
Table III. Predicted Glu-69 Value-of-Information by Baseline Prevalence, Intervention Type, and Value of CBD Avoidance

\begin{tabular}{|c|c|c|c|c|c|}
\hline \multirow{2}{*}{$\begin{array}{l}\text { Baseline } \\
\text { preva- } \\
\text { lence }\end{array}$} & \multirow{2}{*}{$\begin{array}{l}\text { Intervention } \\
\text { type }\end{array}$} & \multicolumn{4}{|c|}{ Value of CBD avoidance } \\
\hline & & $\$ 12,200$ & $\$ 240,000$ & $\$ 1,450,000$ & $\$ 16,300,000$ \\
\hline $0.1 \%$ & Required screen & $-\$ 350(-610,-180)^{a}$ & $-\$ 140(-400,40)$ & $\$ 970(680,1,200)$ & $\$ 15,000(13,000,16,000)$ \\
\hline $0.1 \%$ & Increased LPT frq. & $-\$ 690(-1,000,-410)$ & $-\$ 650(-990,-370)$ & $-\$ 440(-840,-40)$ & $\$ 2,100(-180,5,400)$ \\
\hline $0.1 \%$ & Voluntary screen & $-\$ 330(-560,-180)$ & $-\$ 250(-450,-70)$ & $\$ 210(-280,870)$ & $\$ 5,700(220,14,000)$ \\
\hline $1 \%$ & Required screen & $-\$ 250(-510,-80)$ & $\$ 1,800(1,500,2,100)$ & $\$ 13,000(11,000,14,000)$ & $\$ 150,000(130,000,160,000)$ \\
\hline $1 \%$ & Increased LPT frq. & $-\$ 680(-1,000,-400)$ & $-\$ 290(-790,270)$ & $\$ 1,800(-230,4,700)$ & $\$ 28,000(4,800,61,000)$ \\
\hline $1 \%$ & Voluntary screen & $-\$ 290(-500,-150)$ & $\$ 560(-220,1,700)$ & $\$ 5,100(120,12,000)$ & $\$ 60,000(4,900,140,000)$ \\
\hline $10 \%$ & Required screen & $\$ 710(410,950)$ & $\$ 21,000(19,000,23,000)$ & $\$ 130,000(110,000,140,000)$ & $\$ 1,500,000(1,300,000,1,600,000)$ \\
\hline $10 \%$ & Increased LPT frq. & $-\$ 570(-980,-180)$ & $\$ 3,300(10,8,100)$ & $\$ 24,000(4,100,52,000)$ & $\$ 280,000(54,000,610,000)$ \\
\hline $10 \%$ & Voluntary screen & $\$ 70(-320,630)$ & $\$ 8,000(360,20,000)$ & $\$ 51,000(3,800,120,000)$ & $\$ 570,000(49,000,1,400,000)$ \\
\hline
\end{tabular}

${ }^{a}$ First value indicates expected value in dollars per beryllium worker; range in parentheses indicates $90 \%$ simulation interval as defined by the 5th and 95th percentiles of Monte Carlo simulation results. These values depend on hypothetical models and subjective parameter estimates shown in Table I.

variance. For voluntary screening $98 \%$ of the VOI simulation variance is attributable to uncertainty regarding the removal rate for voluntary screening $\left(w_{\text {volunter }}\right)$. For increased LPT frequency in Glu-69 $9^{+}$ workers, $98 \%$ of the variance in simulated VOI is associated with the risk-reduction efficacy of increased LPT frequency $\left(e_{L P T}\right)$, and $1 \%$ of the variance is associated with the cost of additional LPT testing $\left(C_{L P T}\right)$. Other variables contribute minimally $(<1 \%)$ to the total uncertainty regarding VOI for any of these three programs.

\section{DISCUSSION}

Our analysis indicates that the expected health benefits of a Glu-69-based required screening program for machinists at typical baseline prevalence levels far outweigh the expected financial costs for most health valuations. If an a priori judgment is made that the qualitative social and ethical risks of required screening are too burdensome or if required genetic screening is determined to be illegal, ${ }^{(28)}$ other Glu-69-based risk management programs may be

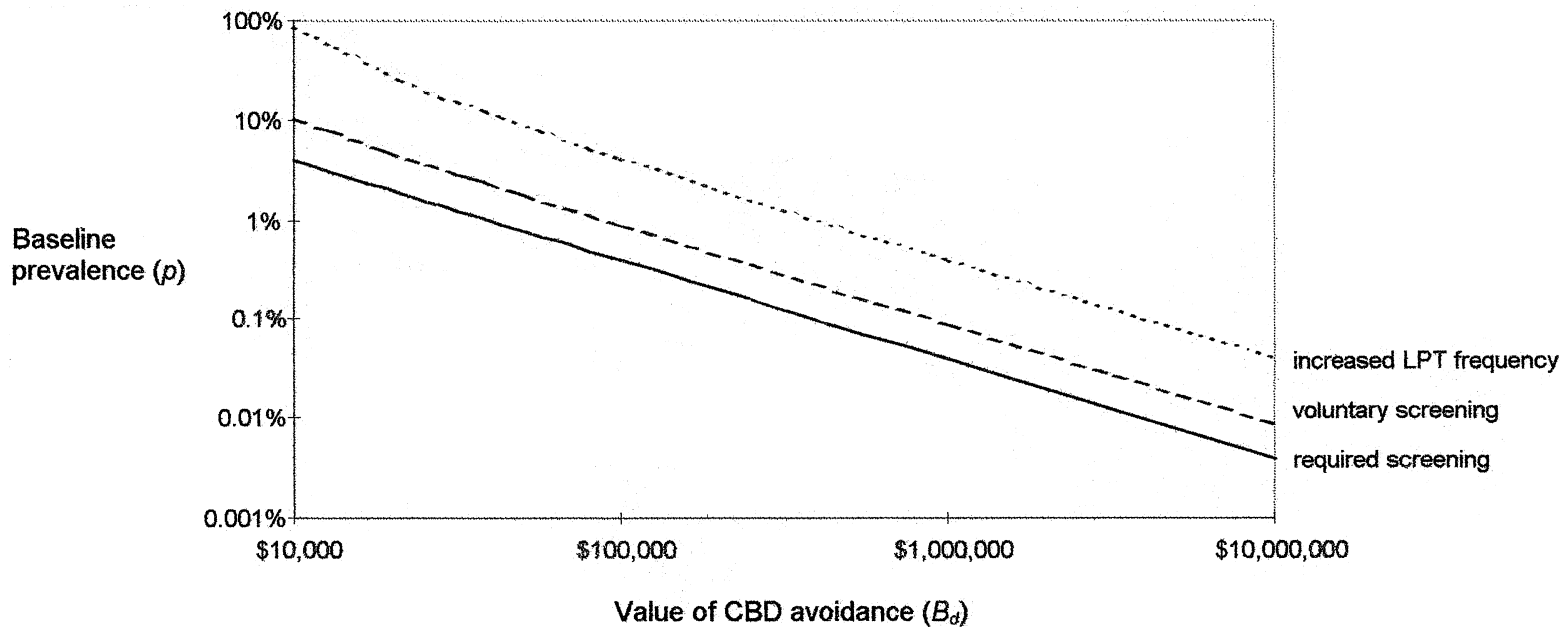

Fig. 2. Values of $p$ and $B_{d}$ that produce an expected VOI of $\$ 0$ for each GLU-69-based intervention. Each line shows the cutpoints for a single intervention type based on possible combinations of the two key parameters, holding all other parameters at their expected values. Regions above each cutpoint line indicate combinations of $p$ and $B_{d}$ expected to result in a positive VOI for that particular GLU-69-based intervention. Regions below each cutpoint line indicate parameter values resulting in a negative expected VOI. All values depend on hypothetical models and subjective parameter estimates shown in Table I. 
more appropriate. Voluntary placement, for example, may be as nearly effective as required screening in reducing disease. The major risk associated with this approach is the possibility that few Glu- $69^{+}$workers will decide to get tested or to avoid placement in berylliumexposed job tasks. However, even if every worker decides to get tested for susceptibility but nobody with Glu- $69^{+}$decides to act on the results, the financial costs are minimal ( $\sim 290 /$ worker). Furthermore, even if only one of every hundred Glu- $69^{+}$applicants decides to remove themselves from exposure, the expected value of the health benefits will still exceed the program costs at health valuations greater than $\$ 500,000$ per CBD case.

Indirect benefits of biomarker development and use exist but have not been evaluated in this model. The identification of the polymorphism and development of the Glu-69 test, for example, may ultimately allow understanding of the mechanisms underlying CBD and thus lead perhaps to better prevention or treatment options. ${ }^{(20)}$ Medical surveillance programs designed around the susceptibility biomarker might similarly lead to new insights regarding CBD etiology. Although it may not be appropriate to include these impacts in deciding whether or not to broadly implement a risk management program based on genetic susceptibility, they are important considerations when targeting biomarker research.

\subsection{Other Risk Management Options}

Not all risk management options have been evaluated here, nor have combinations of the three options. Process changes, personal protective equipment, and limitations on exposure duration are common methods used in industrial hygiene practice to reduce exposure. ${ }^{(29)}$ Indeed, DOE has recently introduced substantial exposure reduction activities as part of a suite of hazard mitigation and communication activities intended to reduce CBD incidence among its workers. ${ }^{(30)}$ Because the dose-response relationship is unclear, whether exposure reductions beyond the current standard will lead to substantially reduced CBD incidence is uncertain. Although it is prudent to assume that further reductions in exposure concentration are likely to reduce disease incidence, for beryllium there is little direct evidence to support or contradict this assumption.

Quantitative dose-response models for berylliuminduced CBD are currently unavailable. Such a model could easily be incorporated into this analysis and would provide a means of estimating the risk reduction and VOI for beryllium exposure controls. Here we chose not to consider the effectiveness of exposure reductions but rather to limit our analysis to the utility of the Glu-69 test at specific baseline CBD prevalence levels. If a Glu-69 genetic screening approach is combined with exposure reductions to all workers, the value of the genetic information depends on the baseline prevalence following the exposure reduction. For example, current CBD prevalence among beryllium machinists has been reported as approximately $10 \%$ for one cohort. ${ }^{(23)}$ If additional exposure controls in these machinists were expected to lead to a $90 \%$ reduction in risk, the new baseline prevalence in this cohort would be $1 \%$, and the expected VOI for the voluntary Glu-69 program would decrease from $\$ 8,000$ to $\$ 560$ per beryllium machinist based on $\$ 240,000$ per CBD case avoided (Table III).

Other programs might be considered in addition to those heightened medical monitoring options evaluated here. For example, the interval between successive LPTs might be changed to other values, and when warranted, other clinical tests could be used to ascertain disease status before the appearance of frank CBD. Although the possibility has not been quantitatively evaluated here, any application of Glu69 genetic screening might indirectly induce some Glu- $69^{+}$beryllium workers to reduce their own beryllium exposures, thereby indirectly increasing the utility of the program. However, the disease reduction benefits of early detection of sensitization or disease are unlikely to be greater than the benefits of required or voluntary preplacement screening, and the financial costs of heightened medical monitoring are relatively high. Nonetheless, heightened medical monitoring targeted to Glu- $69^{+}$workers may be superior to not using Glu-69-based genetic testing at current occupational CBD prevalence rates. In addition, heightened medical monitoring may be the only genetically based intervention available to former beryllium workers.

We have not evaluated the possibility of using multiple genetic polymorphisms in screening. Polymorphisms other than the Glu-69 substitution have been associated with CBD, albeit none have demonstrated as high sensitivity or specificity for predicting CBD. Use of information derived from multiple polymorphisms might increase the sensitivity and specificity of genetically based screening for CBD, and determination of possible genetic linkages would provide insight into whether the Glu-69 polymor- 
phism is causally implicated in disease onset and progression.

Richeldi et al. ${ }^{(2)}$ investigated multiple polymorphisms in the HLA-DPB1 gene after a screening study demonstrated no strong associations with polymorphisms in the HLA-DR and -DQ genes. They report that an aspartic acid-glutamic acid substitution for alanine-alanine at position 55-56 showed significant association with disease $(79 \%$ sensitivity and $59 \%$ specificity). Because individual data were not provided in the publication, however, whether individuals with both polymorphisms exhibited greater disease prevalence was unclear. A concomitant analysis of genetic linkage between Glu-69 and polymorphic sites within HLA-DRB4 and TNFB demonstrated no association with CBD. ${ }^{(2)}$

In a study of genetic polymorphisms associated with beryllium hypersensitivity, Stubbs et al. ${ }^{(31)}$ examined HLA-DRB1, -DRB3, -DRB4, -DRB5, and -DPB1. They observed a significant association between beryllium sensitization and the HLA-DPB1 Glu-69 polymorphism, supporting the observations of Richeldi et $a l^{(2)}$ In addition, they observed that five specific polymorphisms in HLA-DRB1 were associated with susceptibility to beryllium sensitization and that four others within the same gene were associated with protection. Polymorphisms associated with reduced prevalence of sensitization were also observed within HLA-DRB3 and -DRB5. Whether these polymorphisms are associated with development of CBD has not been reported, and genetic linkage between HLA-DP genes and other MHC class II genes is low. ${ }^{(32)}$ Finally, unpublished analyses by Saltini et al. reportedly demonstrate a significant association between CBD and polymorphisms in TNF- $\alpha$; however, the association is reportedly weaker than that observed with Glu-69, ${ }^{(8)}$ and no information is currently available regarding disease prevalence among individuals with both polymorphisms.

\subsection{Social Implications and Ethics of Genetic Screening}

If a decisionmaker decides that the social and ethical risks of required Glu-69 screening should be quantified and weighed against the financial and health risks, Eq. 1 can be expanded to include all of those risks. Because of the difficulty in assigning value to social risks, we have chosen to treat them qualitatively instead of quantitatively in this analysis. For this reason we have also calculated VOI sepa- rately for each risk management option, rather than taking the more typical approach of optimizing before the VOI calculation. ${ }^{(33)}$ We feel that our approach may be more informative to decisionmakers, particularly when they might wish to select a "suboptimal" risk management option based on factors external to the quantitative analysis.

Many authors have pointed out the social and ethical hazards of occupational genetic screening. ${ }^{(34)}$ These hazards include potential loss of employment, benefits, employability, insurability, and privacy for individuals who are genetically susceptible but may not develop disease after beryllium exposure. Because of the potential for these negative impacts, and because the majority of beryllium-exposed Glu- $69^{+}$ individuals will not develop CBD, some beryllium researchers have recommended that Glu-69 not be used for employment screening. ${ }^{(23)}$ We do not intend to belittle these concerns, but when the health risks are substantial the ethical hazards of failing to act are also worthy of consideration. This ethical hazard is heightened in this case by the uncertainty regarding the dose-response relationship for CBD, the absence of a complete cure, and the complications often encountered when using long-term immunosuppressive therapy as is frequently required for CBD. Although the current $2 \mu \mathrm{g} / \mathrm{m}^{3}$ standard is clearly not protective of all workers, further beryllium exposure reductions are not guaranteed to substantially reduce CBD incidence because of the exquisite susceptibility of some individuals. Hence, other options must be considered for use in conjunction with improved exposure controls.

The social risks of taking action on the basis of genetic test results can be mitigated to some extent. For example, DOE has directed that its workers testing positive for sensitization to beryllium be removed from job tasks with known beryllium exposure but retain full employment and benefits for some time after diagnosis. ${ }^{(30)}$ A similar rule could be applied to current workers testing positive for genetic susceptibility to beryllium, though this would increase the costs of the screening program and thereby reduce the expected VOI. In addition, federal legislation disallowing the use of genetic susceptibility tests by insurance companies and preventing inappropriate use of genetic screening by employers has been proposed..$^{(35,36)}$ Although this issue is highly contentious and will likely be discussed for years to come, legal safeguards on the availability and use of genetic information might mitigate some of the concerns raised by bioethicists. For example, employers could hire an inde- 
pendent laboratory to conduct confidential genetic testing and provide confidential counseling to current and prospective beryllium workers. This approach would encourage individuals to get tested by protecting their privacy but would increase screening costs for employers. Revised estimates of the VOI for expanded programs such as these could be obtained by using our model equations.

The success of any voluntary screening program depends on trust. Participation rates can increase over time as a program becomes established and gains credibility. One study reported that over $99.9 \%$ of Maryland parents consented to newborn phenylketonuria screening for medical intervention after the state repealed a required screening law. ${ }^{(37)}$ Anything close to this degree of participation could only be achieved in the occupational setting by carefully addressing the privacy and job security concerns of those tested and through well-conceived risk communication. Although previous surveys found many people reluctant to share genetic information with employers, ${ }^{(38)}$ it is reasonable to believe that some beryllium workers would give informed consent to susceptibility testing and remove themselves from beryllium work on the basis of the results. Given the health risks, we believe that beryllium workers should at least be provided with information about the potential uses of the test.

\subsection{Data Needs and Suggestions for Future Research}

The health valuation for CBD is clearly the most important aspect of this model in terms of impact on the predicted VOI. Readers are likely to have quite different opinions on the most appropriate value of $B_{d}$, and for policy makers to select an appropriate value may be difficult. Although some of these discrepancies could be resolved by COI and WTP studies conducted specifically for $\mathrm{CBD}$, further research is unlikely to resolve basic differences of opinion.

Of the other variables in the model, several stand out as crucial areas for additional research. The voluntary participation rate is unknown as we are unaware of such a genetically based occupational screening model in current use. Even were data available for another genetic screen, how applicable these data would be to the particular case of Glu-69 and CBD is questionable. The specific contextual factors are likely to strongly influence the participation rate for such a program; thus a small-scale pilot study to preliminarily estimate the parameter would be required. We suggest that Glu-69 may be appropriate for such a pilot study, on the basis of the VOI analysis conducted here.

The medical value of higher LPT frequency is also open to question and is represented by a wide range of possibilities in the present model. A detailed quantitative model of beryllium sensitization and CBD progression is needed for this and other aspects of our model, but existing models are mostly qualitative and still open to debate. Future work should include the development and testing of more detailed disease models for CBD, particularly those that take both genetic status and beryllium exposure characteristics into account. For example, we have hypothesized that the sensitivity and specificity rates are independent of baseline CBD prevalence by using a relatively simple model for the interaction of genetics and environment in producing CBD. More cohort study data would allow for the exploration of this model and alternative hypotheses.

\section{CONCLUSIONS}

The Glu-69 genetic biomarker, when used as a required or voluntary screen for occupational placement in beryllium-contaminated areas, is expected to be cost-effective in mitigating CBD in typical cohorts when CBD valuation is above $\$ 100,000$ per case. At lower valuations and lower baseline disease prevalence rates, cost-effectiveness decreases. However, genetic screening has significant ethical and legal implications that may prevent implementation. Voluntary screening may be nearly as cost effective as required screening and is more likely to be accepted by stakeholders, but predicting the participation rate is difficult. Increasing the frequency of the LPT from once a year to twice a year for Glu- $69^{+}$individuals may also decrease disease prevalence at a reasonable cost but is predicted to be less effective than either of the screening programs.

We recommend a dialogue among stakeholders about the use of voluntary Glu-69 testing for beryllium work applicants or existing workers expected to enter areas with known beryllium contamination. If workers' privacy and employability concerns are addressed, voluntary screening can achieve significant disease reduction without undesirable social consequences. Heightened medical monitoring may still be worthwhile, perhaps in conjunction with voluntary screening, but is not as amenable to privacy protec- 
tion as voluntary genetic testing conducted by an outside party.

Many more biomarkers are available today than are actually used in clinical, occupational, or environmental risk management. Highly sensitive and specific biomarkers are clearly valid for practice, but less obvious biomarkers may also offer significant opportunities for disease reduction. Tools such as decision analysis and VOI analysis can assist in discriminating between good and poor biomarker opportunities by providing information about the predicted costs and benefits of using biomarkers in specific applications.

\section{ACKNOWLEDGMENTS}

This manuscript is based on work originally presented at the 1998 American Nuclear Society Topical Meeting on Risk-Based Performance Assessment and Decision Making. ${ }^{(21)}$ Research funding was provided by the Consortium for Risk Evaluation with Stakeholder Participation (CRESP) by Department of Energy Cooperative Agreement \#DE-FCO195EW55084. This support does not constitute endorsement of the views expressed herein.

\section{APPENDIX: ESTIMATION OF POSITIVE AND NEGATIVE PREDICTIVE VALUES FROM SENSITIVITY AND SPECIFICITY RATES}

The relationship between screening biomarkers and disease can be reported in many different ways. Epidemiologists often use positive and negative predictive values $\left(\mathrm{PV}^{+}\right.$and $\left.P V^{-}\right)$as one set of measures indicating the efficacy of binary screening tests for predicting binary disease states. Predictive values can be directly obtained only from cohort studies. Predictive values are simply conditional probabilities $(P r)$ of the test results accurately reflecting the eventual disease state:

$$
\begin{aligned}
& P V^{+}=\operatorname{Pr}\left[D^{+} \mid T^{+}\right] \\
& P V^{-}=\operatorname{Pr}\left[D^{-} \mid T^{-}\right]
\end{aligned}
$$

where $D^{+}$is diseased and $D^{-}$is disease-free, and $T^{+}$is a positive screening result and $T^{-}$is a negative screening result. Sensitivity $(S n)$ and specificity $(S p)$ are different measures of screening efficacy and can be measured in both case-control and cohort studies. $S n$ and $S p$ are the reverse conditional probabilities of $P V^{+}$ and $P V^{-}$:

$$
S n=\operatorname{Pr}\left[T^{+} \mid D^{+}\right]
$$

$$
S p=\operatorname{Pr}\left[T^{-} \mid D^{-}\right]
$$

The proportion of the population screening positive $(f)$ is estimated ${ }^{(39)}$ from $S n, S p$, and the baseline disease risk $(p)$ using Bayes' Rule:

$$
\begin{aligned}
f & =\operatorname{Pr}\left[T^{+}\right] \\
& =\operatorname{Pr}\left[T^{+}, D^{+}\right]+\operatorname{Pr}\left[T^{+}, D^{-}\right] \\
& =\operatorname{Pr}\left[T^{+} \mid D^{+}\right] \operatorname{Pr}\left[D^{+}\right]+\operatorname{Pr}\left[T^{+} \mid D^{-}\right] \operatorname{Pr}\left[D^{-}\right] \\
& =\operatorname{Sn} p+(1-S p)(1-p) .
\end{aligned}
$$

$P V^{-}$and $P V^{+}$are then estimated from $S n, S p, f$, and $p$ in a similar manner:

$$
\begin{aligned}
P V^{+} & =\operatorname{Pr}\left[D^{+} \mid T^{+}\right] \\
& =\operatorname{Pr}\left[D^{+}, T^{+}\right] / \operatorname{Pr}\left[T^{+}\right] \\
& =\operatorname{Pr}\left[T^{+} \mid D^{+}\right] \operatorname{Pr}\left[D^{+}\right] / \operatorname{Pr}\left[T^{+}\right] \\
& =\operatorname{Sr} p / f \\
\operatorname{PV} & =\operatorname{Pr}\left[D^{-} \mid T^{-}\right] \\
& =\operatorname{Pr}\left[D^{-}, T^{-}\right] / \operatorname{Pr}\left[T^{-}\right] \\
& =\operatorname{Pr}\left[T^{-} \mid D^{-}\right] \operatorname{Pr}\left[D^{-}\right] / \operatorname{Pr}\left[T^{-}\right] \\
& =\operatorname{Sp}(1-p) /(1-f) .
\end{aligned}
$$

\section{REFERENCES}

1. A. P. Decaprio, "Biomarkers: Coming Of Age for Environmental Health and Risk Assessment," Environmental Science \& Technology 31(7), 1837-1848 (1997).

2. L. Richeldi, R. Sorrentino, and C. Saltini, "HLA-DPB1 Glutamate 69: A Genetic Marker Of Beryllium Disease," Science 262(5131), 242-244 (1993).

3. R. O. Zerbe, Jr. and D. D. Dively, Benefit-Cost Analysis: In Theory And Practice (HarperCollins College Publishers, New York, 1994).

4. E. Stokey and R. Zeckhauser, A Primer for Policy Analysis (W.W. Norton, New York, 1978).

5. R. T. Clemen, Making Hard Decisions: An Introduction to Decision Analysis, 2nd ed. (Duxbury Press, Belmont, California, 1996).

6. T. K. Takaro, "Beryllium Biomarkers of Exposure, Susceptibility, and Effect," presented at the American Conference on Occupational Health, Boston, 1998.

7. World Health Organization, Environmental Health Criteria 106: Beryllium (World Health Organization, Geneva, Switzerland, 1990).

8. C. Saltini, M. Amicosante, A. Fanchi, G. Lombardi, and L. Richeldi, "Immunogenetic Basis of Environmental Lung Disease: Lessons from the Berylliosis Model," European Respiratory Journal 12, 1463-1475 (1998).

9. D. G. Freiman and H. L. Hardy, "Beryllium Disease: The Relation of Pulmonary Pathology to Clinical Course and Prognosis Based on a Study of 130 Cases from the U.S. Beryllium Case Registry," Human Pathology 1(1), 25-44 (1970).

10. K. Kreiss, M. M. Mroz, L. S. Newman, J. Martyny, and B. Zhen, "Machining Risk of Beryllium Disease and Sensitization with Median Exposures Below $2 \mu \mathrm{g} / \mathrm{m}^{3}$," American Journal of Industrial Medicine 30(1), 16-25 (1996).

11. K. K. Kreiss, "Beryllium," in L. Rosenstock and M. R. Cullen (eds.), Textbook of Clinical Occupational and Environmental Medicine, pp. 734-736, (W.B. Saunders Company, Philadelphia, 1994). 
12. K. Kreiss, M. M. Mroz, B. Zhen, J. W. Martyny, and L. S. Newman, "Epidemiology of Beryllium Sensitization and Disease in Nuclear Workers," American Review of Respiratory Disease 148(4 Pt 1), 985-991 (1993).

13. M. Eisenbud, "The Standard for Control of Chronic Beryllium Disease," Applied Occupational and Environmental Hygiene 13(1), 25-31 (1998).

14. U.S. Department of Energy, "Chronic Beryllium Disease Prevention Program: Notice of Proposed Rulemaking and Public Hearings," Federal Register 63(232), 66,940-66,975 (1998).

15. L. S. Newman, "To $\mathrm{Be}^{2+}$ or Not To $\mathrm{Be}^{2+}$ : Immunogenetics and Occupational Exposure," Science 262(8 October), 197-8 (1993).

16. L. S. Newman, J. Lloyd, and E. Daniloff, "The Natural History of Beryllium Sensitization and Chronic Beryllium Disease," Environmental Health Perspectives 104(Suppl. 5), 937-943 (1996).

17. C. H. Hennekens and J. E. Buring, Epidemiology in Medicine (Little, Brown and Company, Boston, 1987).

18. A. M. Finkel and J. S. Evans, "Evaluating the Benefits of Uncertainty Reduction in Environmental Health Risk Management," Journal of the Air Pollution Control Association 37, 1164-1171 (1987).

19. K. M. Thompson and J. S. Evans, "The Value of Improved National Exposure Information for Perchloroethylene (Perc): A Case Study for Dry Cleaners," Risk Analysis 17(2), 253-271 (1997).

20. M. G. Morgan and M. Henrion, Uncertainty: a Guide to Dealing with Uncertainty in Risk and Policy Analysis (Cambridge University Press, Cambridge, Massachusetts, 1990).

21. S. M. Bartell, R. A. Ponce, T. K. Takaro, G. S. Omenn, R. O. Zerbe, and E. M. Faustman, "Value-of-Information Analyses for Biomarkers: Susceptibility to Chronic Beryllium Disease at US DOE Sites," pp. 205-213 in Proceedings of the Topical Meeting on Risk-Based Performance Assessment and Decision Making, Richland/Pasco, Washington, April 5-8, 1998 (American Nuclear Society, La Grange Park, Illinois, 1998).

22. K. Kreiss, F. Miller, L. S. Newman, Amaize- E. A. Ojo, M. D. Rossman, and C. Saltini, "Chronic Beryllium Disease-From the Workplace to Cellular Immunology, Molecular Immunogenetics, And Back," Clinical Immunology and Immunopathology 71(2), 123-129 (1994).

23. L. Richeldi, K. Kreiss, M. M. Mroz, B. Zhen, P. Tartoni, and C. Saltini, "Interaction of Genetic and Exposure Factors in the Prevalence of Berylliosis," American Journal of Industrial Medicine 32(4), 337-340 (1997).

24. G. Tolley, D. Kenkel, and R. Fabian, Valuing Health for Policy: An Economic Approach (The University of Chicago Press, Chicago, 1994).
25. U.S. Department of Energy, Proposed Chronic Beryllium Disease Prevention Program Rule: Economic Analysis, (Office of Environment, Safety, and Health, Germantown, Maryland, 1998).

26. U.S. Environmental Protection Agency, Guidelines for Preparing Economic Analysis: Review Draft, (U.S. EPA, 1999).

27. K. Kreiss, S. Wasserman, M. M. Mroz, and L. S. Newman, "Beryllium Disease Screening in the Ceramics Industry. Blood Lymphocyte Test Performance and Exposure-Disease Relations," Journal of Occupational Medicine 35(3), 267-274 (1993).

28. Norman-Bloodsaw v. Lawrence Berkeley Laboratory, February 3, 1998 (9th Cir. Report No. WL 39209, 1998).

29. N. A. Ashford, D. B. Hattis, C. J. Spadafor, and C. C. Caldart, Monitoring the Worker for Exposure and Disease (The Johns Hopkins University Press, Baltimore, Maryland, 1990).

30. U.S. Department of Energy, Guidance for Interim Chronic Beryllium Disease Prevention Program (Office of Environment, Safety, and Health Report No. Department of Energy Guidance 440.1-7, 1997).

31. J. Stubbs, E. Argyris, C. W. Lee, D. Monos, and M. D. Rossman, "Genetic Markers in Beryllium Disease," Chest 109(3), 45S (1996).

32. J. M. Baisch and J. D. Capra, "Linkage Disequilibrium within the HLA Complex Does Not Extend to HLA-DP," Scandinavian Journal of Immunology 37, 499-503 (1993).

33. J. W. Pratt, H. Raiffa, and R. Schlaifer, Statistical Decision Theory (The MIT Press, Cambridge, Massachusetts, 1996).

34. N. A. Holtzman, "Medical and Ethical Issues in Genetic screening-An Academic View," Environmental Health Perspectives 104(Suppl 5), 987-990 (1996).

35. E. Marshall, "Clinton Backs Broad Genetic Safeguards," Science 277(5324), 308 (1997).

36. A. A. Love, Vice President Proposes Ban of Genetic Discrimination in the Workplace, AP Wire, January 21, 1998 (Associated Press, 1998).

37. N. A. Holtzman, "Editorial: Genetic Screening and Public Health," American Journal of Public Health 87(8), 1275-1276 (1997).

38. U.S. Department of Labor, Genetic Information and the Workplace, January 20, 1998 (U.S. Department of Labor, Department of Health and Human Services, Equal Employment Opportunity Commission, and Department of Justice, 1998).

39. L. D. Fisher and G. Van Belle, Biostatistics: A Methodology for the Health Sciences (John Wiley \& Sons, New York, 1993). 